$\Rightarrow$ COVID-19

\section{RAAS inhibitors do not increase the risk of COVID-19}

use of ACE inhibitors and ARBs is not associated with increased susceptibility to COVID-19 and does not have harmful effects in patients with COVID-19
According to five new studies, therapy with angiotensin-converting enzyme (ACE) inhibitors or angiotensin-receptor blockers (ARBs) is not associated with an increased risk of infection with severe acute respiratory syndrome coronavirus 2 (SARS-CoV-2) or with an increased risk of severe disease or in-hospital death among patients with COVID-19. These observations are reassuring after concerns had been raised about the possibility that blockers of the renin-angiotensin-aldosterone system (RAAS) could predispose individuals to severe COVID-19. Although the findings from these studies, which were published in NEJM, Lancet and JAMA Cardiology, should be considered exploratory given that all were observational, the findings were consistent across the different patient populations and the different study designs.

Mehra and colleagues used an observational database from 169 hospitals in Asia, Europe and North America to evaluate the association between cardiovascular disease, drug therapy and in-hospital death in 8,910 patients with a confirmed diagnosis of COVID-19. Logistic-regression multivariate analyses showed that age $>65$ years, coronary artery disease, congestive heart failure, cardiac arrhythmia, chronic obstructive pulmonary disease and current smoking were independently associated with an increased risk of in-hospital death. Conversely, use of ACE inhibitors (OR 0.33, 95\% CI 0.20-0.54) or ARBs (OR 1.23, 95\% CI 0.87-1.74) was not associated with a higher risk of in-hospital death.

Mancia and colleagues conducted a population-based, case-control study in Lombardy, Italy, with 6,272 patients with confirmed SARS-CoV-2 infection and 30,759 matched control individuals. Use of ACE inhibitors and ARBs was more frequent among patients with COVID-19 than among control individuals, but conditional logistic-regression multivariate analyses showed no association between these drug classes and the likelihood of SARS-CoV-2 infection or the severity of COVID-19.

Reynolds and colleagues carried out an observational study including data from 12,594 patients who had been tested for SARS-CoV-2 infection in a health network in New York City, NY, USA. Bayesian analyses and propensity-score models showed that previous treatment with antihypertensive drugs (including ACE inhibitors and ARBs) was not associated with either a higher likelihood of a positive test for SARS-CoV-2 infection or a higher risk of severe COVID-19.

Similar findings were obtained by Mehta and colleagues in a cohort of 18,472 patients who had been tested for SARS-CoV-2 infection in the Cleveland Clinic Health System in Ohio and Florida, USA. Overlap propensity score weighting showed no association between the use of ACE inhibitors or ARBs and the likelihood of a positive test (OR 0.97, 95\% CI 0.81-1.15).

Finally, de Abajo and colleagues conducted a case-population study in seven hospitals in Madrid, Spain, including 1,139 patients with a confirmed diagnosis of COVID-19 requiring hospital admission and 11,390 matched controls. Conditional logistic regression analyses showed that the use of RAAS inhibitors was not associated with a higher risk of COVID-19 requiring hospital admission compared with the use of other antihypertensive drugs (adjusted OR 0.94, 95\% CI $0.77-1.15)$. Of note, patients with diabetes mellitus who were taking RAAS inhibitors had a lower risk of COVID-19 requiring hospital admission.

Interestingly, some findings suggest that ACE inhibitors and ARBs have different effects in patients with COVID-19, although the findings are contradictory. Mehra and colleagues found that ACE inhibitor use, as well as statin use, was associated with better survival. By contrast, a secondary exploratory analysis by Mehta and colleagues showed a higher risk of hospitalization among patients with SARS-CoV-2 infection who were taking ACE inhibitors (OR 1.84) or ARBs (OR 1.61). However, the investigators, and the authors of accompanying editorials, caution that these associations should be considered with extreme caution because of potential unmeasured confounding given the observational design of the studies and the small number of patients in the secondary exploratory analysis.

Taken together, these studies suggest that the use of ACE inhibitors and ARBs is not associated with increased susceptibility to COVID-19 and does not have harmful effects in patients with COVID-19.

Irene Fernández-Ruiz

ORIGINAL ARTICLES Mancia, G. et al. Reninangiotensin-aldosterone system blockers and the risk of Covid-19. N. Engl.J. Med. https://doi.org/ 10.1056/NEJMoa2006923 (2020) | Mehra, M. R. et al. Cardiovascular disease, drug therapy, and mortality in Covid-19. N. Engl.J. Med. https://doi.org/10.1056 NEJMoa2007621 (2020)| Reynolds, H. R. et al. Renin-angiotensin-aldosterone system inhibitors and risk of Covid-19. N. Engl.J. Med. https://doi.org/ 10.1056/NEJMoa2008975 (2020) | Mehta, N. et al. Association of use of angiotensin-converting enzyme inhibitors and angiotensin II receptor blockers with testing positive for coronavirus disease 2019 (COVID-19). JAMA Cardiol. https:// doi.org/10.1001/jamacardio.2020.1855 (2020)| de Abajo, F. J. et al. Use of renin-angiotensinaldosterone system inhibitors and risk of COVID-19 requiring admission to hospital: a case-population study. Lancet https://doi.org/ 10.1016/S0140-6736(20)31030-8 (2020) 